\title{
Correction to: Taxonomic, functional and phylogenetic bat diversity decrease from more to less complex natural habitats in the Amazon
}

\author{
William Douglas Carvalho ${ }^{1,2,3}$ (1) Karen Mustin ${ }^{4} \cdot$ Fábio Z. Farneda ${ }^{5}$ Isaí Jorge de Castro ${ }^{6}$ Renato R. Hilário ${ }^{1,3}$.

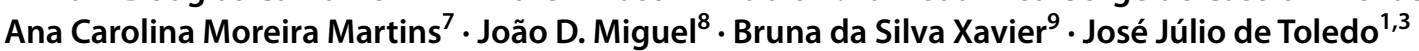

Published online: 27 August 2021

(c) Springer-Verlag GmbH Germany, part of Springer Nature 2021

\section{Correction to: Oecologia https://doi.org/10.1007/s00442-021-05009-3}

Authors would like to correct the error in one of the coauthor name of their publication.

The author name Renato R. Hiláro should be updated as Renato R. Hilário, with the letter "i" in his last name.

The original article has been corrected.

The original article can be found online at https://doi.org/10.1007/ s00442-021-05009-3.

William Douglas Carvalho

wilruoca@hotmail.com

1 Programa de Pós-Graduação em Biodiversidade Tropical, Universidade Federal do Amapá (UNIFAP), Rod. Juscelino Kubitscheck, km 2, Macapá, AP 68903-419, Brazil

2 Programa de Pós-Graduação em Biodiversidade e Meio Ambiente, Universidade Federal da Grande Dourados (UFGD), Rodovia Dourados/Itahum, km 12, Dourados, MS 79804-970, Brazil

3 Laboratório de Ecologia, Departamento de Meio Ambiente e Desenvolvimento, Universidade Federal do Amapá (UNIFAP), Rod. Juscelino Kubitscheck, km 2, Macapá, AP 68903-419, Brazil

4 Programa de Pós-Gradução em Biologia Animal, Departamento de Ecologia, Zoologia e Genética, Instituto de Biologia, Universidade Federal de Pelotas, Pelotas, RS 96010-900, Brazil
5 Department of Ecology and Evolution, Federal University of Santa Maria, Santa Maria, RS 97105-900, Brazil

6 Laboratório de Mamíferos, Instituto de Pesquisas Científicas e Tecnológicas do Estado do Amapá (IEPA), Rodovia JK, Km 10, Macapá, AP 68912-250, Brazil

7 Coordenação de Identificação e Planejamento de Ações Para Conservação (COPAN), Instituto Chico Mendes de Conservação da Biodiversidade (ICMBio), EQSW 103/104, Bloco "C", Complexo Administrativo - Setor Sudoeste, Brasília, DF 70670-350, Brazil

8 Centre for Ecology, Evolution and Environmental Changes and Departamento de Biologia Animal, Faculdade de Ciências, Universidade de Lisboa, 1749-016 Lisbon, Portugal

9 Programa de Pós-Graduação em Ecologia, Universidade Federal do Rio de Janeiro (UFRJ), Av. Carlos Chagas Filho, 373, Rio de Janeiro, RJ 21941-971, Brazil 